\begin{tabular}{|c|c|}
\hline Title & $\begin{array}{l}\text { Combined computational quantum chemistry and classical electrodynamics approach for surface enhanced infrared } \\
\text { absorption spectroscopy }\end{array}$ \\
\hline Author(s) & T akenaka, Masato; Taketsugu, Tetsuy a; Iwasa, Takeshi \\
\hline Citation & $\begin{array}{l}\text { Journal of chemical physics, 152(16), } 164103 \\
\text { https://doi.org/10.1063/1.5143855 }\end{array}$ \\
\hline Issue Date & $2020-04-30$ \\
\hline Doc URL & http:/hdl. handle.net/2115/81094 \\
\hline Rights & $\begin{array}{l}\text { This article may be downloaded for personal use only. A ny other use requires prior permission of the author and AIP } \\
\text { Publishing. This article appeared in Journal of chemical physics } 152(16) 30 \mathrm{~A} \text { pril } 2020,164103 \text { and may be found at } \\
\text { https://ai p.scitation.org/doi } / \text { abs } / 10.1063 / 1.5143855 \text {. }\end{array}$ \\
\hline Type & article \\
\hline File Information & J. Chem. Phys. 152-16_164103.pdf \\
\hline
\end{tabular}

Instructions for use 


\section{Combined computational quantum chemistry and classical electrodynamics approach for surface enhanced infrared absorption spectroscopy}

Cite as: J. Chem. Phys. 152, 164103 (2020); https://doi.org/10.1063/1.5143855

Submitted: 06 January 2020 . Accepted: 02 April 2020 . Published Online: 22 April 2020

Masato Takenaka, Tetsuya Taketsugu (D), and Takeshi Iwasa (D)

\section{ARTICLES YOU MAY BE INTERESTED IN}

Polaritonic normal modes in transition state theory

The Journal of Chemical Physics 152, 161101 (2020); https://doi.org/10.1063/5.0007547

TRAVIS-A free analyzer for trajectories from molecular simulation

The Journal of Chemical Physics 152, 164105 (2020); https://doi.org/10.1063/5.0005078

Large scale and linear scaling DFT with the CONQUEST code

The Journal of Chemical Physics 152, 164112 (2020); https://doi.org/10.1063/5.0005074

\section{Lock-in Amplifiers up to $600 \mathrm{MHz}$}
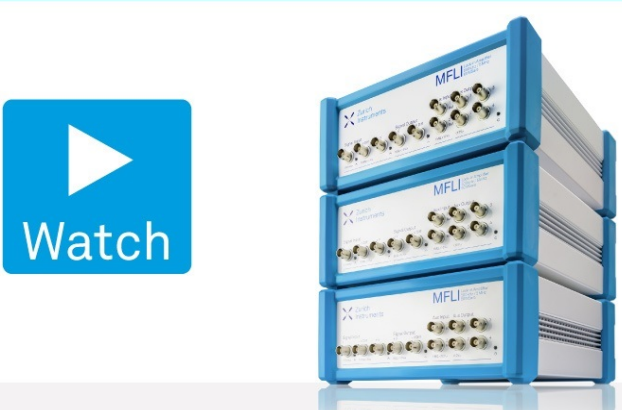

J. Chem. Phys. 152, 164103 (2020); https://doi.org/10.1063/1.5143855 


\title{
Combined computational quantum chemistry and classical electrodynamics approach for surface enhanced infrared absorption spectroscopy
}

\author{
Cite as: J. Chem. Phys. 152, 164103 (2020); doi: 10.1063/1.5143855 \\ Submitted: 6 January 2020 - Accepted: 2 April 2020 • \\ Published Online: 22 April 2020 \\ Masato Takenaka, ${ }^{7}$ Tetsuya Taketsugu, ${ }^{2,3,4}$ (D) and Takeshi Iwasa ${ }^{2,3,4, a)}$ (D) \\ AFFILIATIONS
${ }^{1}$ Graduate School of Chemical Sciences and Engineering, Hokkaido University, Sapporo 060-0810, Japan
${ }^{2}$ Department of Chemistry, Faculty of Science, Hokkaido University, Sapporo 060-0810, Japan
${ }^{3}$ WPI-ICReDD, Hokkaido University, Sapporo 001-0021, Japan
${ }^{4}$ ESICB, Kyoto University, Kyoto 615-8245, Japan
}

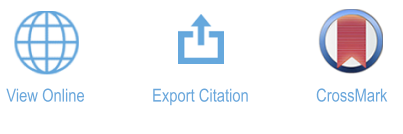

a) Author to whom correspondence should be addressed: tiwasa@sci.hokudai.ac.jp

\begin{abstract}
Surface enhanced spectroscopy, which enhances the signal intensity of molecules on a surface, facilitates the study of molecular properties, even down to a single-molecule level if a scanning probe is used. To realize the full potential of surface enhanced spectroscopy, a clear theoretical understanding is indispensable. However, quantum chemical calculations for surface enhanced spectroscopy are not simple because of the violation of the widely used dipole approximation. The spatial structure of electric near-field in the close proximity of a surface strongly depends on the geometry of the metal nanostructure as well as on the incident wavelength. Therefore, in principle, a universal model for electric near-field cannot exist. To address this issue, we have developed a generalized light-matter interaction model from first-principles quantum chemical calculations by using the multipolar Hamiltonian, in which the spatial structure of the electric field is fully considered. Here, we incorporate computational electrodynamics for surface enhanced infrared (IR) absorption spectroscopy in the model, where electric near-field around a Ag ellipsoid is obtained and used for IR calculations. Furthermore, we have devised a method to successfully reproduce the peak selectivity observed experimentally.
\end{abstract}

Published under license by AIP Publishing. https://doi.org/10.1063/1.5143855

\section{INTRODUCTION}

Infrared (IR) spectroscopy is an indispensable technique to provide fingerprint of various molecules in a non-destructive manner. The applicability of IR spectroscopic techniques is further advanced by using surface plasmon, opening the field of "surface enhanced IR absorption spectroscopy (SEIRAS)." ${ }^{-3}$ Another technique called "surface enhanced IR scattering (SEIRS)" uses a designed surface plasmon as an antenna along with Fano resonance in IR measurements. ${ }^{3-5}$ Furthermore, for microscopic applications, scanning near-field infrared microscopy (SNIM) facilitates nanometer-scale spatial resolution beyond the diffraction limit, ${ }^{6-10}$ as tip-enhanced Raman spectroscopy, which achieves a very high resolution down to a single-molecule level. ${ }^{11,12}$ The surface enhanced techniques not only enhance the signal intensity but also provide an understanding of molecular properties at the nanometer scale. Furthermore, they can be used to study the behavior of a single molecule if combined with metallic probes. To realize the full potential of these techniques, further understanding based on theoretical and computational frameworks is mandatory.

However, it is not simple to obtain surface enhanced IR spectra computationally. In contrast to conventional spectroscopy based on a laser light or propagating light, the applied electric fields are spatially non-uniform in surface enhanced spectroscopy. ${ }^{13,14}$ The 
light-molecule interaction with a propagating light has mostly been studied under the dipole approximation because the wavelength of light in IR or even in the visible region is much larger than the molecular size in conventional experiments. ${ }^{15}$ For non-uniform electric fields, widely available quantum chemistry software cannot calculate the IR spectra because they are based on the dipole approximation. This is even true for just surface spectroscopy such as IR reflection-absorption spectroscopy (IRRAS), where molecules interact with a uniform electric field that is polarized along the surface normal direction, as shown in Fig. 1(a). ${ }^{16}$ In most cases, molecules are assumed to be randomly oriented in a system and interact with incident light. Thus, the IR spectra can be calculated for molecules isotropically interacting with a uniform electric field. However, a recent study has demonstrated that the field gradient plays a crucial role in determining the spectrum corresponding to surface-enhanced Raman scattering (SERS). ${ }^{17}$ In plasmon enhanced spectroscopy, molecules interact with an electric near-field highly localized at the nanometer scale. The importance of the field gradient for understanding SERS is also discussed. ${ }^{18-22}$ In general, the spatial distribution of the electric near-field is not unique. Since there are a variety of techniques for surface enhanced IR measurements as described above, the experimental setups also have a broad spectrum, in which the electric near-field depends on these setups. Thus, we need a more generalized theoretical framework beyond the dipole approximation that is practically tractable with moderate computational cost.
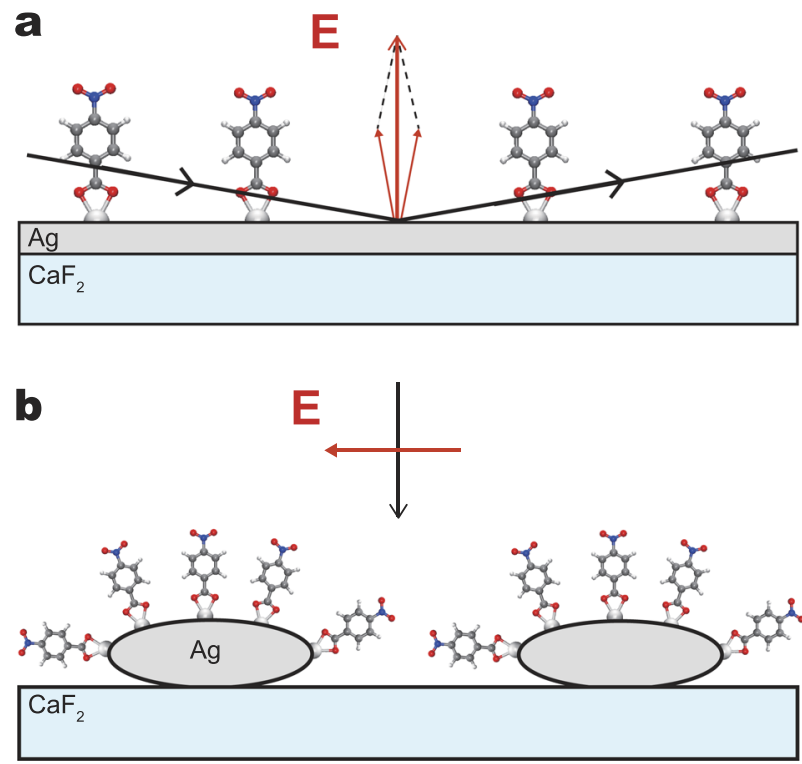

FIG. 1. (a) Schematic of IRRAS, where molecules are adsorbed onto the Ag thin film supported by a substrate. The black arrows show the incident and reflected light beams. Red vectors represent the electric fields of the incident and reflected lights and their superposition, creating an electric field polarized normal to the surface. (b) Schematic of SEIRAS, where the Ag ellipsoid represents the nanoparticles on a substrate and the molecules adsorbed by the nanoparticles. For molecules, red, blue, gray, and white spheres represent $\mathrm{O}, \mathrm{N}, \mathrm{C}$, and $\mathrm{H}$ atoms. The $\mathrm{Ag}$ atom is shown by the silver sphere.
To address this issue, we have developed a computational method to calculate the IR spectra using the multipolar Hamiltonian. ${ }^{23}$ The feasibility of this method was validated by using two cases for IRRAS and SNIM, where we implemented model electric fields. For IRRAS, the electric field was uniform but polarized along the surface normal. For SNIM, we considered the electric field around a metal tip by an electric dipole field. Although the model electric field used here provided a qualitative understanding of surface enhanced spectroscopy, it did not necessarily reproduce the electric field around the metal nanostructure. To this end, numerical calculation of electric field is necessary.

The near-field physics has been intensively studied based on computational electrodynamics, where the Maxwell equations are numerically solved. ${ }^{14}$ Owing to the development of computational electrodynamics software, surface plasmon excitation spectra and/or near-field distribution around nanostructures can now be obtained using routine techniques such as the finite difference time-domain (FDTD) technique and finite element method (FEM). It has been observed that the near-field distribution strongly depends on the nanostructure itself. Thus, the near-field should be properly considered to perform molecular computations for surface enhanced IR spectra.

In this study, we propose a method to compute surface enhanced IR absorption in techniques such as SNIM or SEIRAS by combining quantum chemical computation with classical electrodynamics computation. Electromagnetic calculations were performed to obtain the electric near-field around a metal nanostructure used in experiments, while quantum chemical calculations were performed to compute the electron densities of a molecule in the ground state and the IR signal. The gap between these two methods was bridged by the multipolar Hamiltonian in which an arbitrary electric field could be incorporated.

\section{THEORETICAL FORMULATION: IR ABSORPTION BASED ON THE MULTIPOLAR HAMILTONIAN}

Here, we briefly review our theoretical framework based on the multipolar Hamiltonian in which a numerically obtained electric field can be incorporated. The detailed derivations and discussions on it can be found in our previous paper. ${ }^{23}$ The linear interaction between the $k$ th molecular vibration and electric field in terms of the molecular polarization $\boldsymbol{P}(\boldsymbol{r})$ and electric field $\boldsymbol{E}(\boldsymbol{r})$ is as follows:

$$
V_{\text {int }}^{k}=Q_{k} \int\left(\partial \boldsymbol{P}(\boldsymbol{r}) / \partial Q_{k}\right) \cdot \boldsymbol{E}(\boldsymbol{r}) d \boldsymbol{r} \equiv Q_{k} \partial V_{\text {int }} / \partial Q_{k},
$$

where $Q_{k}$ is the $k$ th normal coordinate and $V_{\text {int }}=\int \boldsymbol{P}(\boldsymbol{r}) \cdot \boldsymbol{E}(\boldsymbol{r}) d \boldsymbol{r}$. To obtain the generalized IR intensity, we consider the expectation value of $V_{\text {int }}^{k}$ using electronic and vibrational wave functions such as $\left|\Psi_{n}\right\rangle\left|v_{i}\right\rangle$

$$
\left\langle v_{1}\left|\left\langle\Psi_{0}\left|V_{\text {int }}^{k}\right| \Psi_{0}\right\rangle\right| v_{0}\right\rangle=\left\langle v_{1}\left|Q_{k}\right| v_{0}\right\rangle \frac{\partial\left\langle\Psi_{0}\left|V_{\text {int }}\right| \Psi_{0}\right\rangle}{\partial Q_{k}} .
$$

Since $\left\langle v_{1}\left|Q_{k}\right| v_{0}\right\rangle$ is constant under the harmonic approximation, ${ }^{24}$ the generalized IR intensity of the $k$ th vibrational mode can be expressed as follows:

$$
I_{k} \propto\left|\frac{\partial A}{\partial Q_{k}}\right|^{2},
$$


where

$$
A \equiv\left\langle\Psi_{0}\left|V_{\text {int }}\right| \Psi_{0}\right\rangle .
$$

Using the exact form of polarization operator, ${ }^{25}$

$$
\hat{\boldsymbol{P}}(\boldsymbol{r})=\sum_{\alpha} e_{\alpha}\left(\hat{\boldsymbol{q}}_{\alpha}-\boldsymbol{R}\right) \int_{0}^{1} d \lambda \delta\left(\boldsymbol{r}-\boldsymbol{R}-\lambda\left(\hat{\boldsymbol{q}}_{\alpha}-\boldsymbol{R}\right)\right),
$$

where $e_{\alpha}$ and $\hat{\boldsymbol{q}}_{\alpha}$ are the charge and the position operator of the $\alpha$ th particle such as an electron or nucleus, and the expectation value of $V_{\text {int }}$ can be expressed as ${ }^{23,26}$

$$
A=\left\langle\Psi_{0}\left|V_{\text {int }}\right| \Psi_{0}\right\rangle=-\int \delta \rho(\boldsymbol{r})(\boldsymbol{r}-\boldsymbol{R}) \cdot \boldsymbol{E}_{\mathrm{eff}}(\boldsymbol{r} ; \boldsymbol{R}) d \boldsymbol{r},
$$

where the electron density difference $\delta \rho(\boldsymbol{r})=\rho(\boldsymbol{r})-\rho^{\text {atom }}(\boldsymbol{r})$ is defined as the difference between the electron density $\rho(\boldsymbol{r})$ of a molecule and the sum of the electron densities of the neutral atoms $\rho^{\text {atom }}(\boldsymbol{r})=\sum_{\alpha} Z_{\alpha} \delta\left(\boldsymbol{r}-\boldsymbol{R}_{\alpha}\right)$ in the molecule, where $Z_{\alpha}$ and $R_{\alpha}$ are the charge and position of the $\alpha$ th nucleus, respectively. The SIESTA code utilizes $\delta \rho$ as a main variable. ${ }^{27}$ By using the electron density difference, the molecular polarization made up of the electron and nuclear parts can be summarized into one term. The detailed discussion can be found in our previous papers.

The effective electric field $\boldsymbol{E}_{\text {eff }}$ is defined as follows:

$$
\boldsymbol{E}_{\mathrm{eff}}(\boldsymbol{r} ; \boldsymbol{R})=\int_{0}^{1} d \lambda \boldsymbol{E}(\boldsymbol{R}+\lambda(\boldsymbol{r}-\boldsymbol{R})),
$$

where $\boldsymbol{R}$ is the center of gravity of a molecule. The integral with respect to $\lambda$ originates from the polarization operator, Eq. (5), where the infinite number of multipoles can be expressed by a compact closed form. By its definition, $\boldsymbol{E}_{\text {eff }}$ is an average electric field between points $\boldsymbol{r}$ and the molecular center $\boldsymbol{R}$. It should be noted that a molecule interacts with $\boldsymbol{E}(\boldsymbol{r})$ itself, not $\boldsymbol{E}_{\text {eff }}$, where the latter appears in our formulation by moving the $\lambda$ integration from the polarization operator to the electric field for numerical applications. The IR intensity given in Eq. (3) is independent of the origin of the coordinate because it is a difference.

For numerical calculations, the effective electric field is expressed in a finite difference form as follows:

$$
\boldsymbol{E}_{\mathrm{eff}}(\boldsymbol{r} ; \boldsymbol{R})=\sum_{i=0}^{M} \frac{1}{M} \boldsymbol{E}\left(\boldsymbol{R}+\frac{i}{M} \boldsymbol{r}\right),
$$

where $M$ is the number of points for the numerical integration. IR intensity is calculated by the square of the absolute value of the derivative of Eq. (6) with respect to the normal coordinates. In the present framework to obtain SEIRAS, the calculation of Eq. (8) is needed only once for every $\boldsymbol{r}$ where $\rho(\boldsymbol{r})$ is nonzero.

\section{COMPUTATIONAL DETAILS}

In this study, we focus on an experiment by Osawaet al., ${ }^{28}$ where IR, IRRAS, and SEIRAS spectra for $p$-nitrobenzoic acid (PNBA) and $m$-nitrobenzoic acid (MNBA) molecules were reported. Figure 1 shows the scheme of IRRAS and SEIRAS, where the Ag thin film and islands are formed on the $\mathrm{CaF}_{2}$ substrate, respectively. The molecules are vertically chemisorbed onto the Ag surface, forming a monolayer. The parameters concerning the IR signals used in the present study are based on our previous paper, ${ }^{23}$ where the numerical accuracy was tested.

\section{A. Molecule: Quantum chemical calculations}

In the IRRAS and SEIRAS experiments, the $\mathrm{CO}_{2}^{-}$group of PNBA and MNBA molecules was vertically chemisorbed onto the Ag surface, while IR spectra were recorded for a PNBA/MNBA$\mathrm{K}$ salt in $\mathrm{KBr}$ pellet. ${ }^{28}$ To simulate this, we replaced one $\mathrm{H}$ atom from the carboxyl groups of these molecules with $\mathrm{Ag}_{3}$ for IRRAS and SEIRAS and with $\mathrm{K}$ for IR to coincide with the experiment. For IRRAS and SEIRAS calculations, we use a very simple model of PNBA-/MNBA- $\mathrm{Ag}_{3}$ with bridge configuration, as shown in Fig. 4, to reduce the computational cost, which is found to be energetically preferred to with atop configurations by $0.63 \mathrm{eV}$ (see Fig. S1). For the adsorption geometries of molecules onto the Ag nanodiscs, PNBA$\mathrm{Ag}_{20}$ are also used to see the preferred adsorption configurations, starting from several initial geometries, where $\mathrm{Ag}_{20}$ of $T_{\mathrm{d}}$ symmetry have four (111) faces. The results for PNBA- $\mathrm{Ag}_{20}$ are shown in the supplementary material (Fig. S2), which also support that the bridging geometry is preferable. It should be noted that the present calculations were performed for neutral systems.

The geometry optimization, generation of the electron density difference, and normal modes analysis were performed using a density functional theory code SIESTA ${ }^{27}$ based on the PerdewBurke-Ernzerhof (PBE) functional $^{29}$ and the numerical basis sets of double-zeta plus polarization (DZP). ${ }^{30}$ The unit cell was calculated to be $25 \times 25 \times 25 \AA$. In this size of the unit cell, there were many points at which the electron density was zero, as schematically shown in Fig. 3. For practical applications, however, a larger unit cell having enough vacuum area would be desirable in order to neglect intermolecular interactions. Only the gamma point was sampled. The electron density differences were generated on a cube file. The mesh cutoff was set to $200 \mathrm{Ry}$. Consequently, the unit cell of the system had $216 \times 216 \times 216$ points with grid spacing of $0.12 \AA$. It should be noted that, in the present framework, the molecular properties such as geometric and electronic structures, as well as the vibrational properties, are not affected by the near-field. In the future, we will investigate the effect of IR near-fields on the molecular structure.

\section{B. Electric field: Electrodynamic calculations}

In IRRAS, it is well known that the electric field is polarized along the surface normal due to the interference between the incident and scattered light. ${ }^{16}$ Thus, electrodynamics calculation was not performed. To simulate IRRAS experiment, a uniform electric field parallel to the molecular axes defined by $\mathrm{Ag}$ and the nearest $\mathrm{C}$ atoms was used. This model has been used in earlier studies to analyze the experimental results in which the derivative of the dipole moment with respect to the normal coordinate was projected to the surface normal.

In contrast, the electric near-field is not generally known in SEIRAS, and thus, we computed the scattered near-field around a Ag nanoparticle. The Ag nanoparticle is modeled by an ellipsoid, as shown in Fig. 2, where the major axes ( $x$ and $y$ ) are parallel to the surface. As shown in Figs. 1(b) and 2, the incident light propagates along the minor axis ( $z$ axis) of the ellipsoid and the electric field is polarized along the $x$ axis. The intensity of the incident electric field was set to $1 \mathrm{~V} / \mu \mathrm{m} \sim 2.7 \times 10^{5} \mathrm{~W} / \mathrm{cm}^{2}$. The scattered electric field around the ellipsoid was calculated by using scuff-em, an open-source software implementation of the boundary-element 

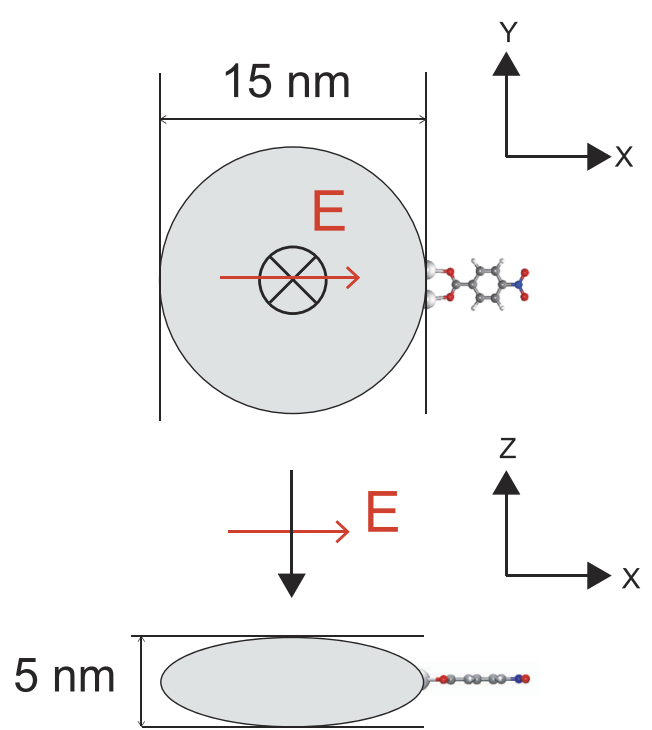

FIG. 2. Schematic of the computational model of SEIRAS. One molecule is adsorbed onto the $\mathrm{Ag}$ ellipsoid with a major axis of $15 \mathrm{~nm}$ and minor axis of $5 \mathrm{~nm}$.

method (BEM) of electromagnetic scattering for the analysis of electromagnetic scattering problems. ${ }^{32}$ The computational cost with the BEM techniques is smaller than FDTD/FEM methods because BEM only computes the electromagnetic field outside the scatterer. This is an advantage for the present purpose because we need to use very fine grids for calculations of the molecule. The computational mesh of the ellipsoid was automatically generated by gmsh. ${ }^{33}$ The Drude model was adopted for the dielectric constant of Ag. ${ }^{34}$ The electric near-fields were calculated for the incident light with wavenumbers of $1000 \mathrm{~cm}^{-1}, 1500 \mathrm{~cm}^{-1}$, and $2000 \mathrm{~cm}^{-1}$ to examine the wavenumber dependence of the peak intensity of SEIRAS. It should be noted that the electric field is only calculated for the Ag ellipsoid without adsorbates, even though the adsorbed molecule is shown in Fig. 2 to indicate the configuration of the adsorbate.
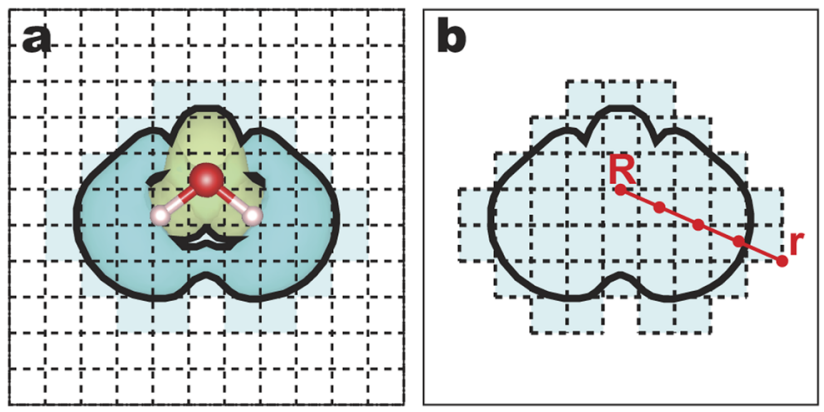

FIG. 3. Schematic of (a) molecular electron density in the unit cell. The points used for further calculations are color shaded. (b) The schematic for the calculation of $E_{\text {eff }}$.

\section{Effective electric field}

The next step is to calculate the effective electric field $\boldsymbol{E}_{\text {eff }}(\boldsymbol{r})$ using Eq. (8). In the present model for SEIRAS, the midpoint of two bridging Ag atoms and the edges of a Ag ellipsoid were matched. The electric near-field was calculated on equally spaced grid points from the center of gravity of the molecular system $\boldsymbol{R}$ to a point $\boldsymbol{r}$. In this study, the number of the grid points was taken as $M=30$, which was used in our previous study as well. ${ }^{23}$ The effective electric field was not calculated at the points where $\delta \rho(\boldsymbol{r})$ was 0 to reduce the computational cost. In the SIESTA code, as shown in Fig. 3(a), there are many such points because the numerical atomic orbital employed has a cutoff at a certain distance from the atom center where the atomic orbital becomes zero, thereby realizing a sparse density matrix for $\mathrm{O}(\mathrm{N})$ calculations. ${ }^{27}$ For the points where $\delta \rho \neq 0$, as shown in the colored area in Fig. 3(b), the effective electric field is calculated by using $M$ points from $R$ to $r$. In the present case, we needed to calculate $\boldsymbol{E}(\boldsymbol{r})$ for about 10000000 points depending on

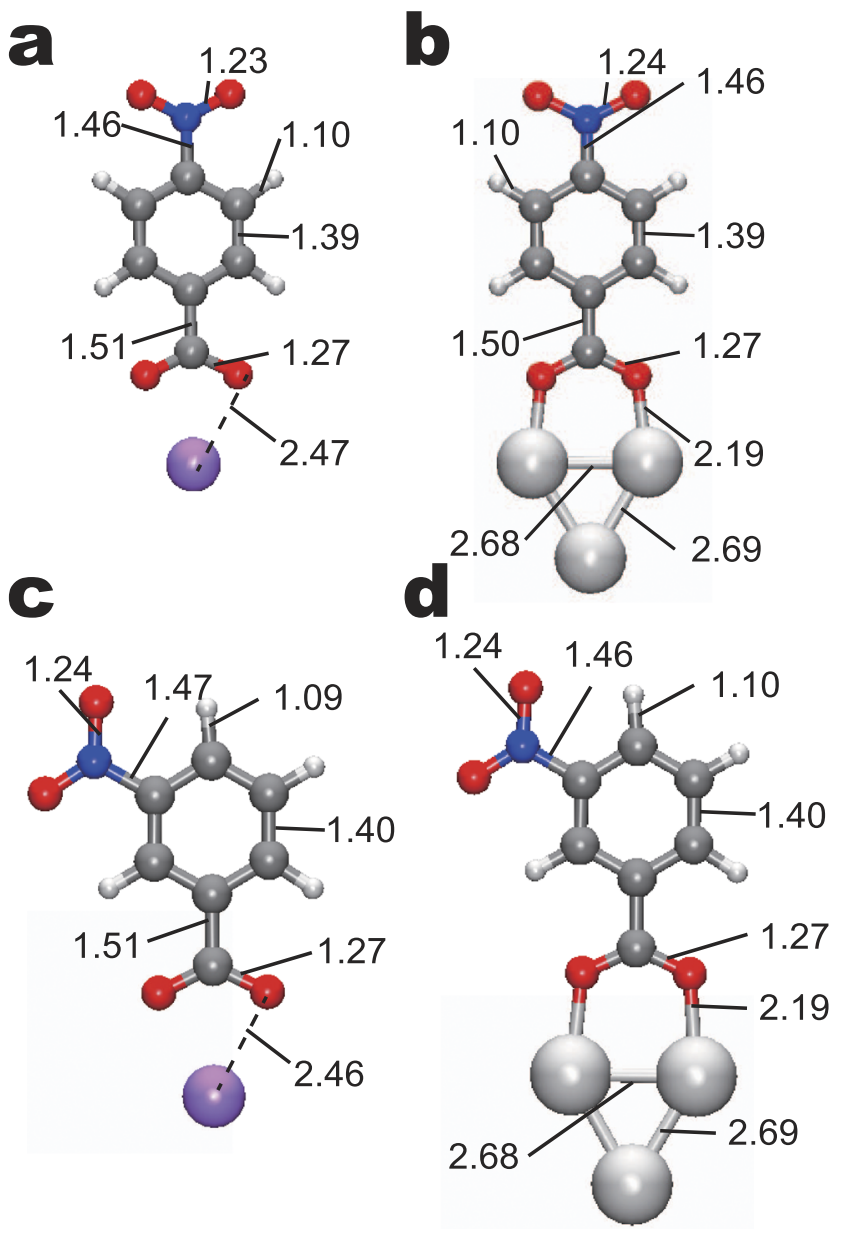

FIG. 4. Optimized structures of (a) PNBA-K, (b) PNBA-Ag ${ }_{3}$, (c) MNBA-K, and (d) MNBA- $\mathrm{Ag}_{3}$, along with the bond lengths in $\AA$. 

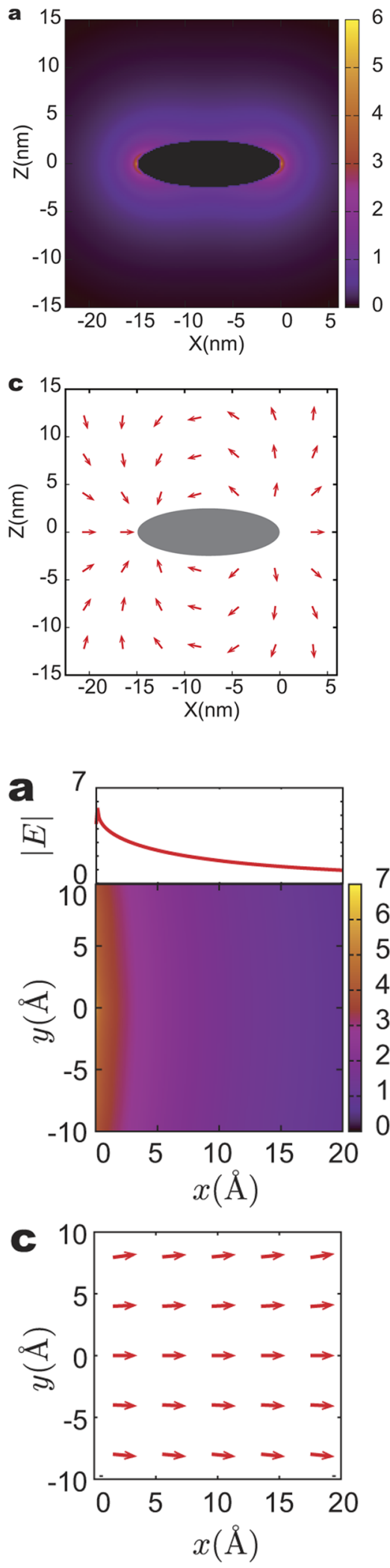

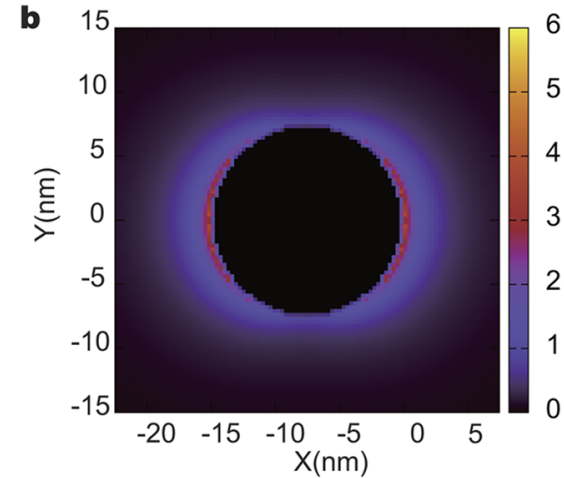

d
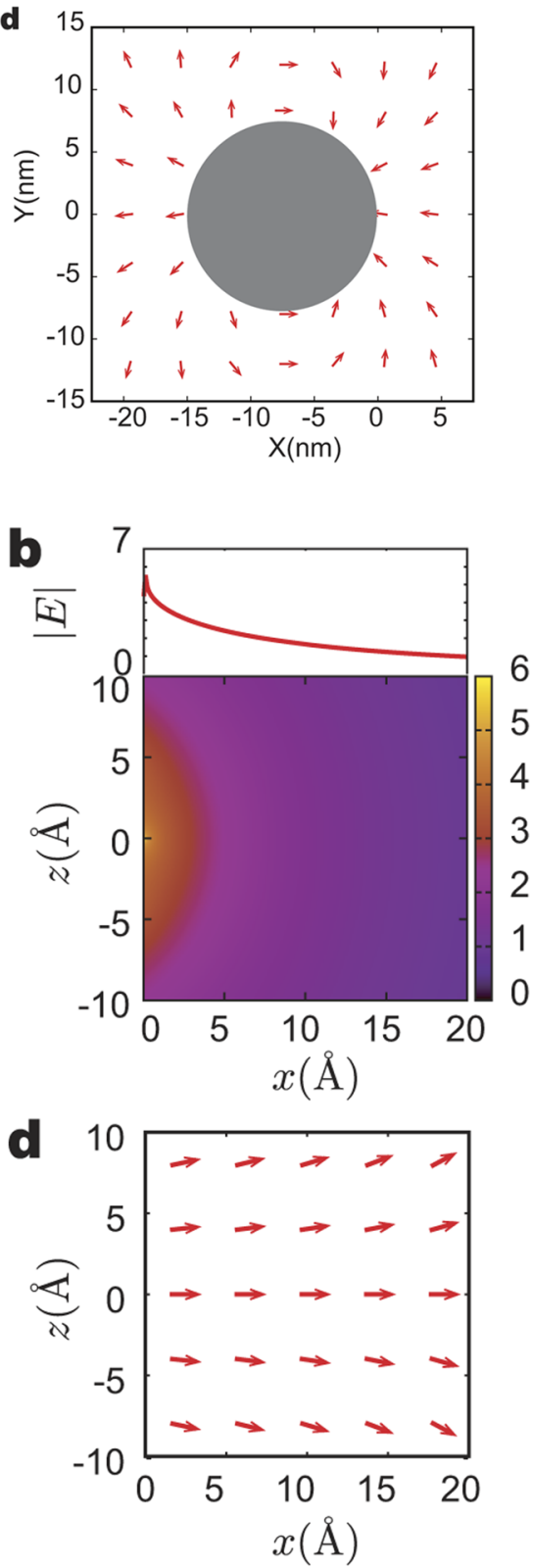

FIG. 5. (a) Side and (b) top views of the relative intensity map of the electric nearfield. The intensity of incident light is considered as 1. (c) Side and (d) top views of the electric fields around the Ag ellipsoid, shown by the red arrows whose intensity is normalized to show the directions. See Fig. 2 for axes.
FIG. 6. Intensity plot and map for (a) the $x y$-plane at $z=0$ and (b) the $x z$-plane at $y=0$. Electric field vectors for (c) the $x y$-plane at $z=0$ and (d) the $x z$-plane at $y=0$. The red vectors are normalized just to show the directions. 
the molecular systems to obtain $\boldsymbol{E}_{\text {eff }}$. These electrodynamics calculations can be independently performed with the parallel efficiency of almost $100 \%$.

\section{IR spectrum}

The normal coordinate derivative of $A$ [Eq. (6)] can be obtained by a matrix transformation from the Cartesian coordinate derivative of $A, \partial A / \partial b_{i}$, where $b_{i}$ is the atomic displacements of the nucleus in the $x, y, z$ coordinates (i.e., $i=0 \ldots 3 N$ ). For the Cartesian coordinate derivative of $A$, each atomic coordinate was displaced in the positive direction from the equilibrium coordinates by 0.04 a.u. as in our previous study. ${ }^{23}$ The electron density difference was calculated for these shifted structures. Finally, the generalized IR intensity was evaluated by Eq. (3).

\section{RESULTS AND DISCUSSION}

The optimized structures of $\mathrm{K}$ - and Ag-adsorbed PNBA and MNBA along with the interatomic distances are shown in Fig. 4. The K/Ag-O distance is the same for these two molecules, indicating a minor effect of the position of the $\mathrm{NO}_{2}$ group on the other parts including the $\mathrm{CO}_{2}^{-}$group. From the Bader charge analysis, the charges of the PNBA/MNBA parts for the neutral systems of PNBA$\mathrm{K}, \mathrm{PNBA}-\mathrm{Ag}_{3}, \mathrm{MNBA}-\mathrm{K}$, and $\mathrm{MNBA}-\mathrm{Ag}_{3}$ are $-1.00,-0.71,-1.00$, and -0.71 , respectively, suggesting that these molecular parts are anionic.

Figure 5 shows the relative intensity and spatial distribution of the near-field around the Ag ellipsoid. The electric field is dipolar. In the relative intensity maps, the incident field strength is considered to be one. It is evident from the relative intensity map [Fig. 5(a)] that the local electric field at the edge of the particle is strong. The intensity of the electric near-field around the Ag ellipsoid is larger than that of the incident field by about five times. However, the enhancement was considered as 70 in the experiment. ${ }^{28}$ Therefore, the observed enhancement in the field strength is about an order of magnitude smaller as compared to that reported in the earlier experiment. In the experimental condition, many metal particles exist, and it is considered that the interaction between them may enhance the electric field intensity due to the overlap of near-fields, i.e., an enhancement at gaps between the metal nanoparticles.

Figure 6 shows the electric field over the region of adsorbed molecule at the molecular scale, where the midpoint of two bridging $\mathrm{Ag}$ atoms is set to $(\mathrm{x}, \mathrm{y}, \mathrm{z})=(0,0,0)$. In other words, the surface of the nanoparticle is considered to be at the origin. Figures 6(b) and 6(d) show that the electric fields are almost uniform, and the intensity gradient exists at the molecular scale, which is about $1 \mathrm{~nm}$. The attenuation of $|E|$ is well fitted by the $-\ln (x)$ function away from the surface, where $x$ is the distance. This is slower than $1 / x$ and much slower than the near-field (longitudinal part) of the electric dipole field, which is proportional to $\left(x^{-3}\right){ }^{14,35}$ Compared to the dipole field, the calculated near-field exhibits much weaker intensity gradient, which indicates that the use of the dipole field is inadequate and, therefore, highlights the importance of computational electrodynamics. The field intensity and distribution are unchanged when the wavenumber of the incident light varies as $1000 \mathrm{~cm}^{-1}$, $1500 \mathrm{~cm}^{-1}$, and $2000 \mathrm{~cm}^{-1}$, i.e., the near-field is independent of the wavenumber. Therefore, the physical enhancement factor (i.e., the enhancement in the electric field) for SEIRAS is independent of the wavenumber used in the present study.

The computed IR, IRRAS, and SEIRAS spectra of PNBA-K and $-\mathrm{Ag}_{3}$ are shown in Fig. 7(a), and the assignment and wavenumbers for representative peaks are listed in Table I. These values are the raw frequencies obtained under harmonic approximation, which generally overestimate the vibrational frequencies. The overestimation could be corrected by taking into account anharmonicity. PNBA-K and PNBA- $\mathrm{Ag}_{3}$ show similar vibrational frequencies and coordinates. Peaks 1 and 2 in the IR spectra disappear in the case of IRRAS and SEIRAS. The derivative of the dipole moment with respect to the $k$ th normal coordinate, $\partial \vec{\mu} / \partial Q_{k}$, for each vibrational mode is shown by a red arrow in Fig. 7 (b). This suggests that the peaks disappear because $\partial \vec{\mu} / \partial Q_{k}$ is almost orthogonal to the electric field. On the contrary, the derivative of the dipole moment for peaks 3 and 4 are

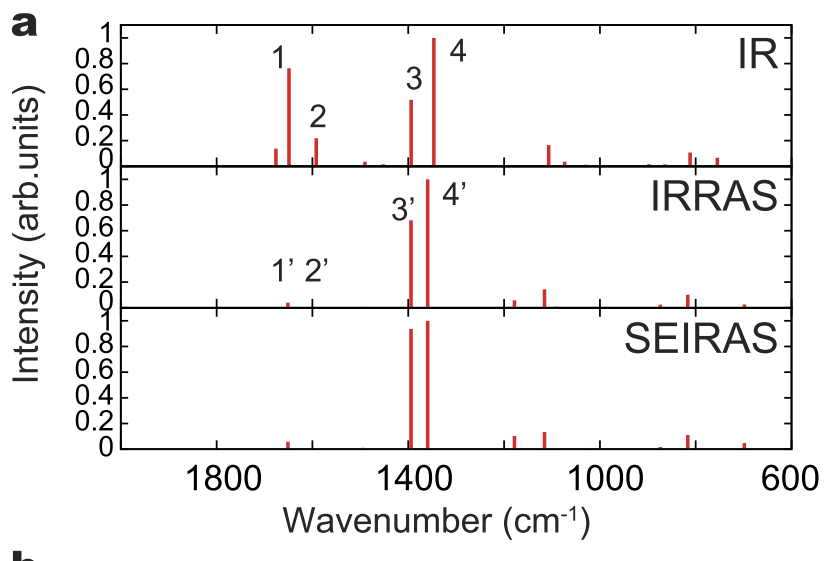

b
1

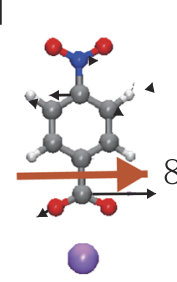

1

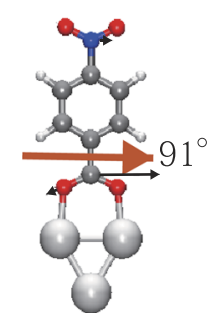

2

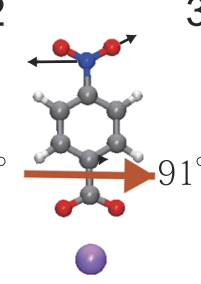

3

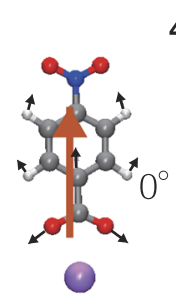

2'

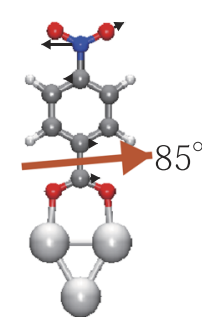

4

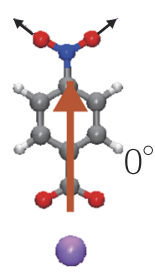

$3^{\prime}$

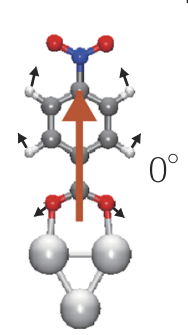

$4^{\prime}$

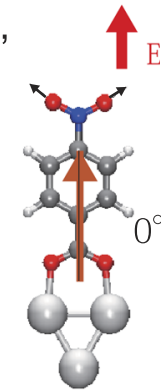

FIG. 7. (a) IR and IRRAS and SEIRAS spectra of PNBA-K and PNBA-Ag ${ }_{3}$, respectively, from top to bottom. (b) Vibrational modes of peaks $1-4$ and $1^{\prime}-4^{\prime}$. Red arrows show the normal coordinate derivatives of the dipole moment vectors, and the angles relative to the electric field are shown alongside. 
TABLE I. Calculated and experimental wavenumbers of vibrational modes of PNBA-K and PNBA- $\mathrm{Ag}_{3}$ in $\mathrm{cm}^{-1}$. In the experiment, ${ }^{28}$ two peaks with higher wavenumbers are missing due to the selection rule for IRRAS and SIERAS.

\begin{tabular}{lcccccc}
\hline \hline & \multicolumn{2}{c}{ Calc. PNBA- } & & \multicolumn{3}{c}{ Expt. $^{28}$} \\
\cline { 2 - 3 } \cline { 7 - 8 } Assignment & $\mathrm{K}$ & $\mathrm{Ag}_{3}$ & & $\mathrm{IR}$ & IRRAS & SEIRAS \\
\hline $\mathrm{V}_{s}\left(\mathrm{NO}_{2}\right)$ & 1347 & 1359 & & 1345 & 1351 & 1338 \\
$\mathrm{~V}_{s}\left(\mathrm{COO}^{-}\right)$ & 1394 & 1394 & & 1383 & 1388 & 1387 \\
$\mathrm{~V}_{a s}\left(\mathrm{NO}_{2}\right)$ & 1592 & 1605 & & 1515 & & \\
$\mathrm{~V}_{a s}\left(\mathrm{COO}^{-}\right)$ & 1649 & 1625 & & 1577 & & \\
\hline
\end{tabular}

parallel to the electric field, and these peaks are observed in the spectra corresponding to IRRAS and SEIRAS. The peak disappearance is not caused by changing the counter-cation/substrate from $\mathrm{K}$ to $\mathrm{Ag}_{3}$. To show this, IR, IRRAS, and SEIRAS of PNBA-Ag are shown

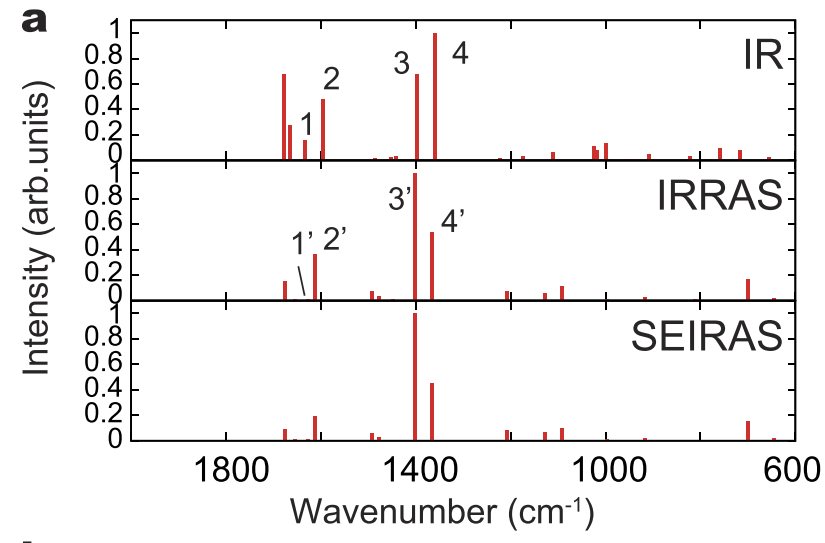

b

1

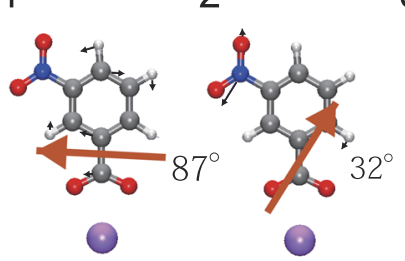

3

$1^{\prime}$

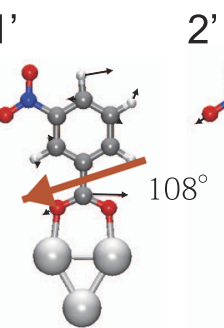

$2^{\prime}$

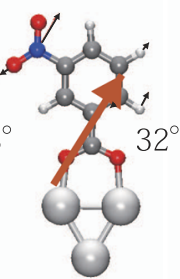

3'

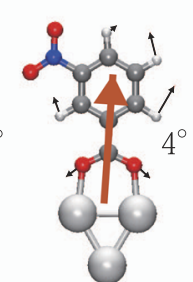

4
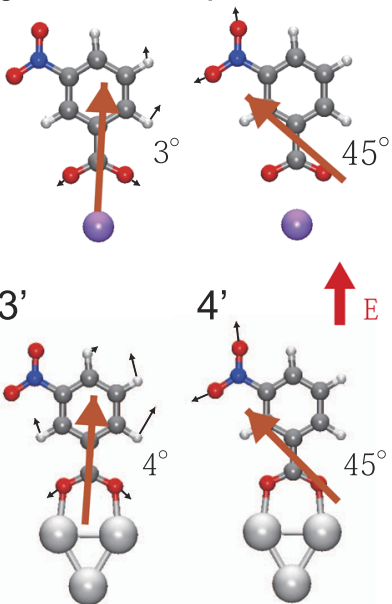

FIG. 8. (a) IR and IRRAS and SEIRAS spectra of MNBA-K and MNBA-Ag, respectively, from top to bottom. (b) Vibrational modes of peaks $1-4$ and $1^{\prime}-4^{\prime}$. Red arrows show the normal coordinate derivatives of the dipole moment vectors, and the angles relative to the electric field are shown alongside.
TABLE II. Integrated intensity ratio of the peaks $3^{\prime}$ and $4^{\prime}$ from Figs. 7 and 8.

\begin{tabular}{lllllll}
\hline & & \multicolumn{2}{c}{ PNBA } & & \multicolumn{2}{c}{ MNBA } \\
\cline { 3 - 4 } \cline { 5 - 7 } \multicolumn{2}{c}{ Peak number } & $3^{\prime}$ & $4^{\prime}$ & & $3^{\prime}$ & $4^{\prime}$ \\
\hline \multirow{2}{*}{ IRRAS } & Expt. $^{28}$ & 1.0 & 0.9 & & 1.0 & 0.6 \\
& Calc. $_{2}^{*}$ SEIRAS & 1.0 & 1.4 & & 1.0 & 0.5 \\
& Expt. $^{28}$ & 1.0 & 1.3 & & 1.0 & 1.4 \\
& Calc. & 1.0 & 1.1 & & 1.0 & 0.4 \\
\hline \hline
\end{tabular}

in Fig. S3 where the same system is used to compare these spectra. Our computational results are in excellent agreement with the experimental results. ${ }^{28}$ Accordingly, we infer that the present method can be used to study the surface selection rule, where only the vibrational modes whose dipole-moment derivative vector is perpendicular to the surface are allowed for IRRAS and SEIRAS. Our method is based on the first-principles calculations, and thus it can be used to analyze more complex molecules without any symmetry, whose adsorption configurations are unknown.

Similarly, the result for MNBA is shown in Fig. 8. Here, peak 1 corresponds to an antisymmetric stretching vibrational mode of $\mathrm{CO}_{2}$. In the case of IRRAS and SEIRAS, this peak disappears because the vibrational mode is tilted by $87^{\circ}$ and $108^{\circ}$ with respect to the electric field for MNBA-K and $\mathrm{MNBA}-\mathrm{Ag}_{3}$, respectively. In the experiment, this missing peak is assigned to the same $\mathrm{CO}_{2}$ vibrational mode. Again, the computational result agrees with Osawa's experimental result. ${ }^{28}$

Table II compares the intensity of the strong peaks 3 and 4 (Fig. 7) obtained by IRRAS and SEIRAS. They represent symmetric stretching vibrations of $\mathrm{CO}_{2}$ and $\mathrm{NO}_{2}$, respectively. For PNBA, the ratio of the intensities of peaks 3 and 4 in the experiment is reversed in SEIRAS as compared to that in IRRAS. Furthermore, the same trend is observed for MNBA. It is considered that the intensity gradient affects the intensity ratio because the peak of $\mathrm{CO}_{2}$ is stronger in SEIRAS than in IRRAS. The integrated intensity ratio was evaluated by the spectral area from the previous report. ${ }^{28}$ It may be noted that the computed intensity ratios do not match with the experimental results. The intensities should be decreased and increased for the peaks 3 and 4, respectively, from IRRAS to SEIRAS because the vibration motions are close to and far from the metal surface. Therefore, the most probable origin of the discrepancy is the lack of total absorbance of the system including the metal. Meanwhile, another reason may be attributed to the use of one metal atom for the adsorption on a surface. The use of the surface itself can provide more accurate and quantitative results, but this necessitates treating the surface with periodic boundary conditions, which forms the future scope of this study.

\section{CONCLUSION}

We have developed a computational method for analyzing surface enhanced IR absorption by incorporating electrodynamic calculations in our quantum chemical model based on multipolar Hamiltonian. The practicality of this model was validated by examining IR, IRRAS, and SEIRAS spectra of small molecules. For SEIRAS, 
the electric near-field around the Ag ellipsoid has been calculated by the boundary element method. The local electric near-field is strong around the edge of the ellipsoid, and the field is polarized along the surface normal. The computed spectra based on these electric fields have reproduced the IR spectra as well as the selection rule of IRRAS and SEIRAS. The present method is based on first-principles calculation and, therefore, exhibits immense potential to investigate unknown and more complex molecules if metal nanostructures used to enhance the electric field are characterized.

\section{SUPPLEMENTARY MATERIAL}

See supplementary material for further structures of PNBA/MNBA- $\mathrm{Ag}_{3}$ and PNBA- $\mathrm{Ag}_{20}$, and IR, IRRAS, and SEIRAS of PNBA- $\mathrm{Ag} / \mathrm{Ag}_{3}$ and MNBA-Ag.

\section{ACKNOWLEDGMENTS}

The authors thank Professor Masatoshi Osawa for fruitful discussions. M.T. is grateful to the Institute for Quantum Chemical Exploration for the IQCE fellowships for Young Scientists. T.I. acknowledges the financial support from JSPSKAKENHI, Grant No.17K14428. The calculations were partly performed at the Research Center for Computational Science, Okazaki, Japan. This work was partly supported by the Elements Strategy Initiative of MEXT, Grant No.JPMXP0112101003, the Photo-excitonix Project in Hokkaido University, and JST CREST, Japan, Grant No. JPMJCR1902.

\section{REFERENCES}

${ }^{1}$ A. Hartstein, J. R. Kirtley, and J. C. Tsang, "Enhancement of the infrared absorption from molecular monolayers with thin metal overlayers," Phys. Rev. Lett. 45, 201-204 (1980).

${ }^{2} \mathrm{M}$. Osawa, "Dynamic processes in electrochemical reactions studied by surfaceenhanced infrared absorption spectroscopy (SEIRAS)," Bull. Chem. Soc. Jpn. 70, 2861-2880 (1997).

${ }^{3}$ F. Neubrech, C. Huck, K. Weber, A. Pucci, and H. Giessen, "Surface-enhanced infrared spectroscopy using resonant nanoantennas," Chem. Rev. 117, 5110-5145 (2017).

${ }^{4}$ F. Neubrech, A. Pucci, T. W. Cornelius, S. Karim, A. García-Etxarri, and J. Aizpurua, "Resonant plasmonic and vibrational coupling in a tailored nanoantenna for infrared detection," Phys. Rev. Lett. 101, 157403 (2008).

${ }^{5}$ P. Biagioni, J.-S. Huang, and B. Hecht, "Nanoantennas for visible and infrared radiation," Rep. Prog. Phys. 75, 024402 (2012).

${ }^{6} \mathrm{~B}$. Knoll and F. Keilmann, "Near-field probing of vibrational absorption for chemical microscopy," Nature 399, 134-137 (1999).

${ }^{7}$ R. Hillenbrand, T. Taubner, and F. Keilmann, "Phonon-enhanced light-matter interaction at the nanometre scale," Nature 418, 159-162 (2002).

${ }^{8}$ T. Taubner, R. Hillenbrand, and F. Keilmann, "Nanoscale polymer recognition by spectral signature in scattering infrared near-field microscopy," Appl. Phys. Lett. 85, 5064-5066 (2004).

${ }^{9}$ M. B. Raschke, L. Molina, T. Elsaesser, D. H. Kim, W. Knoll, and K. Hinrichs, "Apertureless near-field vibrational imaging of block-copolymer nanostructures with ultrahigh spatial resolution," ChemPhysChem 6, 2197-2203 (2005).

${ }^{10}$ E. Bründermann and M. Havenith, "SNIM: Scanning near-field infrared microscopy," Annu. Reports Sect. C 104, 235 (2008).

${ }^{11} \mathrm{~J}$. Steidtner and B. Pettinger, "Tip-enhanced Raman spectroscopy and microscopy on single dye molecules with $15 \mathrm{~nm}$ resolution," Phys. Rev. Lett. 100, 236101 (2008).
${ }^{12}$ R. Zhang, Y. Zhang, Z. C. Dong, S. Jiang, C. Zhang, L. G. Chen, L. Zhang, Y. Liao, J. Aizpurua, Y. Luo, J. L. Yang, and J. G. Hou, "Chemical mapping of a single molecule by plasmon-enhanced Raman scattering," Nature 498, 82-86 (2013).

${ }^{13}$ S. A. Maier, Plasmonics: Fundamentals and Applications (Springer Science \& Business Media, 2007).

${ }^{14}$ L. Novotny and B. Hecht, Principles of Nano-Optics (Cambridge University Press, Cambridge, 2012).

${ }^{15}$ G. C. Schatz and M. A. Ratner, Quantum Mechanics in Chemistry, dover ed. (Dover Publications, Inc., Mineola, NY, 2002).

${ }^{16} \mathrm{~F}$. Hoffmann, "Infrared reflection-absorption spectroscopy of adsorbed molecules," Surf. Sci. Rep. 3, 107 (1983).

${ }^{17}$ M. Takase, H. Ajiki, Y. Mizumoto, K. Komeda, M. Nara, H. Nabika, S. Yasuda, H. Ishihara, and K. Murakoshi, "Selection-rule breakdown in plasmon-induced electronic excitation of an isolated single-walled carbon nanotube," Nat. Photonics 7, 550-554 (2013).

${ }^{18}$ J. K. Sass, H. Neff, M. Moskovits, and S. Holloway, "Electric field gradient effects on the spectroscopy of adsorbed molecules," J. Phys. Chem. 85, 621-623 (1981).

${ }^{19}$ E. J. Ayars, H. D. Hallen, and C. L. Jahncke, "Electric field gradient effects in Raman spectroscopy," Phys. Rev. Lett. 85, 4180-4183 (2000).

${ }^{20}$ P. K. Jain, D. Ghosh, R. Baer, E. Rabani, and A. P. Alivisatos, "Near-field manipulation of spectroscopic selection rules on the nanoscale," Proc. Natl. Acad. Sci. U. S. A. 109, 8016-8019 (2012).

${ }^{21}$ D. V. Chulhai and L. Jensen, "Determining molecular orientation with surfaceenhanced Raman scattering using inhomogenous electric fields," J. Phys. Chem. C 117, 19622-19631 (2013).

${ }^{22}$ L. Meng, Z. Yang, J. Chen, and M. Sun, "Effect of electric field gradient on subnanometer spatial resolution of tip-enhanced Raman spectroscopy," Sci. Rep. 5, 9240 (2015).

${ }^{23}$ T. Iwasa, M. Takenaka, and T. Taketsugu, "Generalized theoretical method for the interaction between arbitrary nonuniform electric field and molecular vibrations: Toward near-field infrared spectroscopy and microscopy," J. Chem. Phys. 144, 124116-1-124116-8 (2016).

${ }^{24}$ P. C. Cross, E. B. Wilson, Jr., and J. C. Decius, Molecular vibrations: The Theory of Infrared and Raman Vibrational Spectra (Dover Publications, Inc., NY, 1955).

${ }^{25}$ D. P. Craig and T. Thirunamachandran, Molecular Quantum Electrodynamics: An Introduction to Radiation-Molecule Interactions (Dover, Mineola, NY, 1998).

${ }^{26}$ T. Iwasa and K. Nobusada, "Nonuniform light-matter interaction theory for near-field-induced electron dynamics," Phys. Rev. A 80, 043409 (2009).

${ }^{27}$ J. M. Soler, E. Artacho, J. D. Gale, A. García, J. Junquera, P. Ordejón, and D. Sánchez-Portal, "The SIESTA method for $a b$ initio order-N materials simulation," J. Phys.: Condens. Matter 14, 2745 (2002).

${ }^{28}$ M. Osawa, K.-I. Ataka, K. Yoshii, and Y. Nishikawa, "Surface-enhanced infrared spectroscopy: The origin of the absorption enhancement and band selection rule in the infrared spectra of molecules adsorbed on fine metal particles," Appl. Spectrosc. 47, 1497-1502 (1993).

${ }^{29}$ J. P. Perdew, K. Burke, and M. Ernzerhof, "Generalized gradient approximation made simple," Phys. Rev. Lett. 77, 3865 (1996).

${ }^{30} \mathrm{~J}$. Junquera, Ó. Paz, D. Sánchez-Portal, and E. Artacho, "Numerical atomic orbitals for linear-scaling calculations," Phys. Rev. B 64, 235111 (2001).

${ }^{31}$ T. Iwasa, K. Horiuchi, M. Shikishima, Y. Noguchi, S. Nagaoka, and A. Nakajima, "Theoretical investigation of a titanium-aniline complex with and without an alkyl chain," J. Phys. Chem. C 115, 16574-16582 (2011).

${ }^{32}$ M. T. H. Reid and S. G. Johnson, "Efficient computation of power, force, and torque in BEM scattering calculations," IEEE Trans. Antennas Propag. 63, 35883598 (2015).

${ }^{33} \mathrm{C}$. Geuzaine and J.-F. Remacle, "Geometric numerical integration for complex dynamics of tethered spacecraft," Int. J. Numer. Methods Eng. 79, 1309-1331 (2009).

${ }^{34}$ P. B. Johnson and R. W. Christy, "Optical constants of the noble metals," Phys. Rev. B 6, 4370-4379 (1972).

${ }^{35}$ J. D. Jackson, Classical Electrodynamics, 3rd ed. (Wiley, New York, 1999). 